identify another mode of exposure to HIV. She was a patient in the dental office from 1987 to 1989 and received prophylaxis and restorative fillings under local anesthesia.

DNA sequence analysis showed that her HIV strain had a high degree of similarity to that of the dentist and five other infected patients. The precise events resulting in HIV transmission in this practice remain unknown. Unlike the other five infected patients, this patient had neither dental extractions nor root canal therapy. Opportunities for injuries to the dentist were limited. However, exposure of this patient to the dentist's blood cannot be ruled out (eg, related to use of the anesthetic syringe).

Approximately 1,100 patients of the dentist are known to have been tested for HIV This dental practice in Florida remains the only documented instance of HIV transmission from a healthcare worker to patients.

FROM: Centers for Disease Control and Prevention. M M WR 1993;42:320-331.

\section{CDC Reports No HIV Infection in Patients Treated by HIV-Infected HCWs}

The CDC recently reported the results of investigations of 19,036 persons treated by 57 HIV-infected healthcare workers (HCWs). No seropositive persons were reported among 11,529 patients tested from practices of $46 \mathrm{HCWs}$, including 23 dentists and dental students, 12 physicians and medical students, seven surgeons and obstetricians, and four others. For the remaining $11 \mathrm{HCWs}, 7507$ patients were tested and 92 seropositive patients were identified. Follow-up investigations have been completed for $86(94 \%)$ of these 92 patients; eight patients were documented to be infected before receiving care from the $\mathrm{HIV}$-infected $\mathrm{HCW}$; 54 had established risk factors for HIV; 19 may have had opportunities for HIV exposure (ie, exchange of sex for drugs or money) ; and five had no risks identified. Investigations are in progress for six patients of two HCWs.

The CDC reports that the risk for transmission of a bloodborne pathogen from an HCW to a patient is associated with the circulating titer of the pathogen in the blood, the procedure performed, techniques and infection control precautions used, and the medical condition of the HCW. The cluster of six cases associated with the dental practice in Florida represents the only documented instance of HIV transmission from an HCW to patients. The data presented in this report of 57 investigated practices are consistent with previous assessments that the risk for HIV transmission from an infected $\mathrm{HCW}$ to patients during invasive procedures is small and can be reduced with appropriate use of infection control precautions.

In an editorial on HIV-infected surgeons and dentists that appeared in the April 14, 1993, issue of the Journal of the A merican M edical A ssociation, Drs. Ban Mishu and William Schaffner of the Vanderbilt University School of Medicine, Nashville, Tennessee, noted that although the lookback studies done to date have been important in establishing that the overall risk to patients from HIV-infected HCWs is low, these studies are unlikely to detect the infrequent highly infectious practitioner who might pose a substantially higher risk to patients. Further, they note that although the Florida dental case has been characterized as an aberration because no other cases of transmission from $\mathrm{HCW}$ to patients have been reported, in fact, it rather closely resembles providerto-patient transmission of $\mathrm{HBV}$, which often produces clusters of cases in the absence of obvious infection control lapses.

FROM: Centers for Disease Control and Prevention. MMWR 1993;42:320-331; Mishu B, Schaffner W. JAMA 1993;269:1843-1844.

\section{OSHA Creates Office of Occupational Health Nursing}

The U.S. Department of Labor announced that a new office of Occupational Health Nursing has been established to underscore the major role nurses play in striving for safe and healthful workplaces. OSHA estimates that there are 23,000 practicing occupational health nurses nationwide, representing the largest group of healthcare providers at the nation's worksites.

The new office will help the agency in developing standards, surveillance policies, and implementing education and training activities, as well as assist with field investigations and evaluation of employees' medical care and injury/illness surveillance records.

\section{New Recommendation Calls for HBV Vaccination for All College Students}

The American College Health Association (ACHA) recently recommended that all colleges and universities require hepatitis B vaccine for healthcare students and strongly urge vaccination for all college students with special attention to those at highest risk of infection. Health officials have urged colleges and universities to conduct campaigns to educate students 
about the disease and encourage them to be vaccinated. University representatives have said that because many universities don't have the money to pay for vaccination, it is unlikely they will mandate such vaccinations prior to admission.

The American Academy of Pediatrics and the U.S. Public Health Service's Advisory Committee on Immunization Practices currently recommend hepatitis B immunization for all infants and older children, adolescents, and adults at increased risk of infection. Both groups also encourage routine immunization of all adolescents.

\section{Latex Allergies Reported by OR Nurses}

Latex glove sensitivities were reported by 369 (21\%) of the 1,738 operating room (OR) nurses recently surveyed at an Association of Operating Room Nurses (AORN) annual convention. The findings of this questionnaire survey were reported by Dr. Stephanie Zaza, medical epidemiologist at CDC, at this year's Epidemic Intelligence Service (EIS) conference in Atlanta, Georgia. Those nurses reporting reactions to latex were more likely than those without such reactions to have a history of other allergies, asthma, and chronic illnesses. The reported symptoms included itching $(86 \%)$, rash $(54 \%)$, dermatitis (51\%), hives (13\%), facial swelling (4\%), and wheezing (1\%). Symptoms were reported most frequently after contact with latex gloves, sterile or nonsterile, powdered or unpowdered. These findings add to the mounting evidence that potentially severe latex glove allergies appear to be increasing.

Although many of the reactions appeared to be related to individual risk factors for allergies, additional studies are needed to determine whether specific types of gloves could be particularly allergenic.

\section{New Technique to Detect Small Amounts of HIV in Blood Cells}

It is now possible to detect HIV DNA or RNA in a small number of blood cells using a highly sensitive new assay technique developed by Dr. Bruce Patterson and colleagues at Northwestern University Medical School in Chicago. The technique involves the use of polymerase chain reaction (PCR) to amplify HIV nucleic acids within cells, tagging them with a fluorescent probe and analyze them using flow cytometry, This new assay allows researchers to quantify the number of HIV-infected cells in peripheral blood and to isolate them for further characterization. This new assay is able to identify HIV genetic material in as few as one in 10,000 blood cells compared with previous assays that could detect HIV in only one in ten blood cells.

FROM: Patterson BK et al. Science 1993;260:976979.

\section{PCR Test Kit for Detection of Chlamydia Awaits FDA Clearance}

The use of polymerase chain reaction (PCR), a new tool in molecular biology, may soon be adding the detection of chlamydia to its list of applications. Roche Molecular Systems, Inc., a division of Hoffmann-La Roche, has submitted a PCR test kit for detection of chlamydia to the FDA and is awaiting clearance. Hoffmann-Ia Roche recently acquired full rights to PCR technology from Cetus Corp. and now owns all technical, patent, and manufacturing rights to all known and future uses for PCR. PCR testing is currently available for HIV, HTLV-I and HTLV-II, Borrelia burgdorferi (Lyme disease), mycobacteria, and paternity testing through Roche Biomedical Laboratories. PCR research technology also has yielded promising results in amplifying the genetic sequences responsible for cystic fibrosis and Duchenne's muscular dystrophy.

\section{EcoliO157:H7 Transmission Reported in Minnesota Daycare Centers}

Although only two reports of Escherichia coli O157:H7 outbreaks in child daycare centers had been published previously, the Minnesota Department of Health identified 68 cases of E coli $0157: H 7$, including 29 cases at nine daycare centers with evidence of person-to-person transmission in all nine daycare centers. Cases of $\mathrm{E}$ coli $\mathrm{O} 157: \mathrm{H} 7$ infection were identified by interviewing parents of infected children under 5 years of age from July 1988 through December 1989. If the child attended a daycare after onset, stool cultures were obtained from other children in attendance and their parents were interviewed. If there was presumptive evidence of ongoing $E$ coli 0157:H7 transmission in a facility, all preschool children were excluded from attending daycare centers until two consecutive stool cultures were negative. There was no evidence of further transmission at centers where children were excluded temporarily until two consecutive stool cultures were negative.

The authors concluded that the number of unrecognized daycare outbreaks in the United States may 